\title{
BMJ Open Reducing health inequalities through general practice: protocol for a realist review (EQUALISE)
}

\author{
John Alexander Ford (D) , ${ }^{1}$ Anna Gkiouleka, ${ }^{1}$ Isla Kuhn, ${ }^{2}$ Sarah Sowden (D) , ${ }^{3}$ \\ Fiona Head, ${ }^{4}$ Rikke Siersbaek (D) , ${ }^{5}$ Clare Bambra, ${ }^{6}$ Rebecca R Harmston, ${ }^{7}$ \\ Sukaina Manji, ${ }^{8}$ Annie Moseley, ${ }^{9}$ Geoff Wong ${ }^{10}$
}

To cite: Ford JA, Gkiouleka A, Kuhn I, et al. Reducing health inequalities through general practice: protocol for a realist review (EQUALISE). BMJ Open 2021;11:e052746. doi:10.1136/ bmjopen-2021-052746

- Prepublication history and additional supplemental material for this paper are available online. To view these files, please visit the journal online (http://dx.doi.org/10.1136/ bmjopen-2021-052746)

Received 23 April 2021 Accepted 13 May 2021

Check for updates

(C) Author(s) (or their employer(s)) 2021. Re-use permitted under CC BY-NC. No commercial re-use. See rights and permissions. Published by BMJ.

For numbered affiliations see end of article.

Correspondence to Dr John Alexander Ford; jf653@medschl.cam.ac.uk

\section{ABSTRACT}

Introduction Healthcare organisations recognise the moral imperative to address inequalities in health outcomes but often lack an understanding of which types of interventions are likely to reduce them. This realist review will examine the existing evidence on the types of interventions or aspects of routine care in general practice that are likely to decrease or increase health inequalities (ie, inequality-generating interventions) across cardiovascular disease, cancer, diabetes and chronic obstructive pulmonary disease.

Methods and analysis Our realist review will follow Pawson's five iterative stages. We will start by developing an initial programme theory based on existing theories and discussions with stakeholders. To navigate the large volume of literature, we will access the primary studies through the identification of published systematic reviews of interventions delivered in general practice across the four key conditions. We will examine the primary studies included within each systematic review to identify those reporting on inequalities across PROGRESS-Plus categories. We will collect data on a range of clinical outcomes including prevention, diagnosis, follow-up and treatment. The data will be synthesised using a realist logic of analysis. The findings will be a description and explanation of the general practice interventions which are likely to increase or decrease inequalities across the major conditions.

Ethics and dissemination Ethics approval is not required because this study does not include any primary research. The findings will be integrated into a series of guiding principles and a toolkit for healthcare organisations to reduce health inequalities. Findings will be disseminated through peer-reviewed publications, conference presentations and user-friendly summaries. PROSPERO registration number CRD42020217871.

\section{INTRODUCTION}

In the last decades, population health has significantly improved at least in high-income countries. ${ }^{1}$ However, it seems that this benefit is not proportionately distributed across groups within societies. Social inequalities in life expectancy, morbidity, mortality and their risk factors remain a persistent problem ${ }^{2}$ which is expected to worsen during and after

\section{Strengths and limitations of this study}

- A key strength of this study is that it will assimilate and synthesise a breadth of data across four conditions and different manifestations of health inequalities.

- A further strength is that the programme theory will be used to develop guidance and a toolkit for practitioners and policy makers in collaboration with patient representatives at every state of the research.

- The two-stage approach to accessing the breadth of literature means that there is a possibility that some studies may be missed.

the COVID-19 pandemic. ${ }^{3}$ Recent reports show that chronic conditions such as cancer, heart disease, stroke and chronic obstructive pulmonary disease (COPD) are driving health inequalities in life expectancy. ${ }^{4}$ Specifically, in England, the gap in life expectancy between the least and most deprived areas is 9.4 years for men and 7.4 years for women, ${ }^{5}$ while the aforementioned conditions were responsible for two-thirds of premature mortality in the country in $2017 .{ }^{6}$ Although these inequalities are driven by wider determinants of health, such as employment, education and income, national healthcare systems have a vital role in defining their range and effects. ${ }^{7}$ In particular, general practice is key to reducing health inequalities. Previous research exploring cancer pathways found that inequalities tend to arise due to late presentation and diagnosis in general practice, rather than the diagnosis to treatment pathway in secondary care. ${ }^{8}$

However, understanding which are the most effective strategies for reducing health inequalities represents a challenge for national and international public health organisations. Besides routine healthcare that often fails to meet the needs of disadvantaged groups and increases health inequalities, there are also health interventions that may 
decrease or increase inequalities. For example, workplace interventions to increase employee control or participation, fiscal policies, such as tobacco price increases and low agency interventions (ie, individuals only require a small amount of effort to benefit) are likely to reduce inequalities. ${ }^{9}$ However, media campaigns and workbased smoking bans tend to increase inequalities across socioeconomic groups and so do interventions which require high agency (ie, individuals require considerable personal resources to benefit). The latter are examples of what we call intervention-generated inequalities (IGIs) ${ }^{10}$ and refer to inequalities emerging from interventions which although they improve health overall, they increase inequalities by disproportionally benefitting advantaged groups or unintentionally negatively affecting disadvantaged groups. ${ }^{11-15}$ Moreover, the extent to which interventions increase or decrease inequalities may also vary over time. For example, research assessing inequalities in smoking found that population-based smoking interventions may increase inequalities in the short-term as they benefit the most-affluent in society; however, over the long-term reduce inequalities as the benefit moves more towards lower socioeconomic groups. ${ }^{16}$

Healthcare services, which are often the first point of contact for vulnerable individuals, are estimated to contribute $15 \%-43 \%$ to health outcomes. ${ }^{7}$ In the UK, the National Health Service (NHS) acknowledges both a moral imperative to address systematic, unfair differences in health outcomes and a financial rationale with socioeconomic inequalities costing the NHS $£ 4.8$ billion/ year in hospitalisations alone. ${ }^{17}$ The NHS long-term plan promises a 'more concerted and systematic approach to reducing health inequalities' and about $£ 1$ billion additional funding for more deprived areas, ${ }^{18}$ with primary care being asked to develop specific plans to reduce health inequalities. ${ }^{19}$ However, in the context of complex and often contradictory evidence as described above, the NHS still lacks a thorough understanding of which types of interventions are likely to reduce inequalities ${ }^{20}$ and paradoxically which may inadvertently increase inequalities. ${ }^{21}$ This realist review study will examine the existing evidence on interventions and aspects of routine care in general practice that are likely to increase or decrease health inequalities and will provide healthcare organisations with an evidence synthesis and guiding principles on what should be commissioned and how for the reduction of health inequalities.

\section{Rationale}

The last systematic review on health service interventions to reduce inequalities was published 23 years ago based on articles published 25-35 years ago. ${ }^{22}$ The study focused on how to reduce inequalities but did not account for IGIs. The authors concluded that a multidisciplinary approach using a combination of strategies was more likely to reduce inequalities. However, the evidence base and the healthcare organisational landscape has changed substantially over the past 25 years.
An important development concerns the PROGRESSPlus conceptual framework for different dimensions of inequalities. ${ }^{23}$ Proposed by the Campbell and Cochrane Collaborations, the framework encourages researchers to take a more systematic approach to equity by categories of disadvantaged groups-place of residence, race/ ethnicity/culture/language, occupation, gender/sex, religion, education, socioeconomic status, social capital, plus characteristics related to discrimination (eg, age and disability), features of relationships (eg, parents who smoke) and time-dependent relationships (eg, being a carer).

Attwood and colleagues ${ }^{14}$ reviewed primary-care-based physical activity interventions using the PROGRESSPlus categories and identified 24 randomised controlled trial (RCT) studies in which differential effects of one or more PROGRESS-Plus criteria were reported, with gender $(\mathrm{n}=22)$ and age $(\mathrm{n}=16)$ being the most common, followed by race $(n=4)$, education $(n=3)$, social capital $(n=3)$ and socioeconomic status $(n=3)$. The authors found mixed effects for gender, and some evidence of improved physical activity for patients with higher baseline social support. An additional review by Terens and colleagues $^{24}$ on quality improvement strategies to reduce inequalities in diabetes care using the PROGRESS-Plus criteria showed that a quality improvement strategy improves care for older and less educated people, and African Americans. Moreover, Capewell and Graham ${ }^{25}$ studied the role of cardiovascular disease prevention in the reduction of health inequalities. They compared the evidence about the effectiveness of a 'high-risk approach'; where patients are categorised and treated according to risk, with 'population-wide programmes', like smoking bans and found that the former is more likely to increase inequalities compared with the latter. Similarly, Durand and colleagues ${ }^{15}$ reviewed the impact of shared decisionmaking across primary and secondary care on health inequalities and found that such interventions were likely to benefit disadvantaged groups and therefore reduce inequalities.

Although important, this evidence is disparate and limited as it emerges from reviews focused on RCTs. Broadening the search to include non-RCT interventional or other studies will allow additional relevant interventions to emerge and be integrated into useable guidance. For example, a review of reviews shows that while the impact of social determinants of health interventions is generally unclear, there is evidence that housing and working environment interventions reduce health inequalities. ${ }^{26} \mathrm{In}$ this context, our study aims to provide healthcare systems and general practices with up-to-date, extended and integrated evidence on interventions that decrease or increase health inequalities with details on who is most and least likely to benefit, in what circumstances, why and how, as well as specific guiding principles to commission general practice effectively to reduce inequalities. Additionally, the review will produce findings that can be applied internationally to guide general practice in a variety of 
settings by uncovering specific contextual factors which are generally present in primary care settings and trigger mechanisms causing inequalities to increase or decrease.

\section{Objectives}

Our main objective is to explore what types of interventions or aspects of routine care in general practice increase or decrease inequalities in outcomes of cardiovascular disease, cancer, diabetes or COPD, for whom, why, in what circumstances and how. Based on this evidence, we aim further to develop guidance and a toolkit for healthcare decision makers about how best to tackle health inequalities through general practice services.

\section{METHODS AND ANALYSIS}

Existing research indicates that the interventions and routine care delivered in general practice are complex and context-specific, with a range of impacts on inequalities in different settings. To account for this complexity, we will conduct a realist review, aiming to complete it by June 2022. In contrast to systematic reviews which seeks to draw conclusions on whether an intervention works or not, realist methods not only seek to understand which specific elements of interventions work, but also for which groups does it work, how does it work and why. ${ }^{10}{ }^{27}$ Specifically it draws on a context-mechanism-outcome (CMO) logic of analysis to develop context-dependent findings and outputs. The review will be conducted through the following steps.

\section{Identifying existing theories}

We will start by identifying existing theories that explain how, for whom, why and in what circumstances interventions delivered in general practice may increase or decrease health inequalities. To do this, we will first scope the literature on IGIs, starting with existing systematic reviews. ${ }^{9} 101427$ The scoping of the literature will be exploratory based on informal methods like snow-balling and citation tracking ${ }^{28}$ to ensure that it will inform the search strategy without limiting its scope. Second, we will consult key content experts and third, we will refine our findings through iterative discussions within the project team to synthesise an initial programme theory. In line with the realist methodology, the initial programme theory as agreed among the different stakeholders will be broad enough to allow generalisability and guide our literature search. ${ }^{27}$

\section{Literature search and screening}

In our review, we will undertake an electronic search of MEDLINE, EMBASE, CINAHL, PsycINFO, the Web of Science and the Cochrane library to identify systematic reviews of interventions delivered in general practice across cardiovascular disease, cancer, diabetes or COPD. Our search strategy is available in online supplemental appendix 1. The identified titles and abstracts will be screened for eligibility by two reviewers and disagreements and unclear studies will be resolved through discussion. Any disagreements will be resolved through discussion. Our inclusion and exclusion criteria are found below.

\section{Inclusion criteria}

- Reviews that use a comprehensive search strategy and appropriate quality appraisal tool.

- Interventions which target cardiovascular disease, cancer, diabetes or COPD or their risk factors, namely, smoking, hypertension, diet, exercise and cholesterol.

- Interventions delivered in general practice.

- Clinical outcomes relating to the type of care (acute, chronic and preventative), function of care (diagnosis, screening and prevention, follow-up and continuity, treatment) and domain of care (effective, efficient, timely, patient-centred and safe).$^{29}$

- Studies reporting on the differential effectiveness of care/intervention across groups or interventions targeted at disadvantaged groups.

- Studies undertaken in high-income countries, as defined by the Organisation for Economic Co-operation and Development (OECD), with no language restrictions.

\section{Exclusion criteria}

- Drug effectiveness or efficacy studies.

- Review articles superseded by more comprehensive reviews.

- Reviews on children, mental health or disease areas not listed above.

Next, the full text of the included primary studies in each of the reviews will be screened by two reviewers according to the following criteria:

\section{Inclusion criteria}

- Interventions which report clinical outcomes by socioeconomic group, or other PROGRESS-Plus criteria, relating to the type of care (acute, chronic and preventative), function of care (diagnosis, screening and prevention, follow-up and continuity, and treatment) and domain of care (effective, efficient, timely, patient-centred and safe).

- Interventional studies or interventions targeted at disadvantaged groups.

- General practice interventions targeting cardiovascular disease, cancer, diabetes or COPD or their risk factors, namely, smoking, hypertension, diet, exercise and cholesterol.

- Clinical outcomes (eg, mortality and myocardial infarction) or clinical measures of risk factors (eg, change in Hb1Ac and blood pressure).

- Experimental study design.

- Studies undertaken in high-income countries, as defined by the OECD, with no language restrictions.

\section{Exclusion criteria}

- Drug effectiveness or efficacy studies.

More searches will be undertaken, if we require more data to develop and test certain subsections of 
the programme theory. This will allow us to integrate useful evidence from non-RCT studies which are usually excluded from systematic reviews. For each additional search, the project team will set inclusion and exclusion criteria and follow the same screening processes as for the initial search. Where applicable, we will follow the search strategies developed by Booth and colleagues. ${ }^{30}$

\section{Article selection}

Documents will be selected based on relevance (ie, whether data can contribute to theory building and/ or testing) and rigour (ie, whether the methods used to generate the relevant data are credible and trustworthy). To illustrate how we will operationalise rigour, if relevant data have been generated using an RCT, then the trustworthiness of the data would be considered to be greater if the trial meets current quality criteria for rigour. Documents may still be included even if judged to be of limited rigour, as we will also be making an overall assessment of rigour at the level of the programme theory ${ }^{31}$ following the same decisionmaking process as outlined above.

\section{Data extraction}

The included papers will be uploaded to NVivo and sections of texts relating to contexts, mechanisms and their relationships to outcomes will be coded by AG. Codes will be inductive (ie, created to categorise data reported in included studies), deductive (ie, created in advance of data extraction as informed by the initial programme theory) and retroductive (ie, created based on an interpretation of data to infer what the hidden causal forces might be for outcomes). A random sample of $10 \%$ will be independently and additionally coded by JF and/or GW to improve consistency and quality. Key study characteristics, such as included patients, interventions and outcomes by socioeconomic group or other PROGRESS-Plus criteria, will be extracted using an Excel pro forma. Each new element of data will be used to refine the theory if appropriate, and as the theory is refined, included studies will be re-scrutinised for data relevant to the revised theory that may have been missed initially.

\section{Data synthesis}

Data will be analysed in light of the initial programme theory following current realist review quality and publication standards. ${ }^{32}$ We will use interpretive crosscase comparison to understand and explain how and why observed outcomes have occurred. As part of data synthesis, we will use a series of questions addressing the relevance and rigour of content, interpretation of meaning, as well as interpretations about CMOconfigurations and the initial programme theory within and across documents. Theory driven data synthesis will allow us to move beyond the identification of intervention effects towards the causal mechanisms operating behind specific interventions and ultimately towards suggestions regarding the ways interventions can be more useful.

\section{Developing guidance and a toolkit for practitioners and policy makers}

The finalised programme theory, as it will emerge from the data synthesis, will be used to develop guidance and a toolkit for practitioners and policy makers. First, the research team will develop an initial guidance document and toolkit similar to previous work ${ }^{33}$ which will then be refined during a 1-day deliberative workshop with the expert group, research team and key stakeholders (eg, NHS England, groups which represent disadvantaged communities). We are confident that the engagement of key stakeholders encouraged by realist methodology will benefit the outcomes and ensure their effectiveness within service delivery settings.

\section{Patient and public involvement}

Patient and public involvement (PPI) in the delivery of the research will be key. We will have three PPI representatives as part of the Research Team. Our PPI team members will ensure that a patient and public voice is maintained throughout the research and will contribute to the design, interpretation of the findings and dissemination activities. Furthermore, we will recruit a diverse range of individuals to attend expert group meetings and the Deliberative workshop.

\section{Ethics and dissemination}

Ethics approval is not required because this study does not include any primary research.

Our dissemination strategy will capitalise on the stakeholder networks that we will establish during the research. We will consult with our expert panel to identify the main stakeholders for dissemination across different audiences and accordingly develop tailored materials for each audience, namely, primary care policy makers and commissioners, general practitioners, members of the public, third-sector organisations and researchers.

To ensure that the findings have maximum impact we will produce a number of outputs tailored to the needs of the targeted audiences. We will produce a set of guiding principles aimed at all general practice services which will help decision makers and commissioners to understand the likely impact of their services on health inequalities and offer evidence-based suggestions for the tailoring or modification of services. The guidelines will further be integrated in a toolkit with greater detail and worked examples. The main target audience of this toolkit will be national, regional and local healthcare organisations. Additionally, the findings from the review will be submitted for publication to an open-access high-impact peer-reviewed journal for the academic audience. Our final project report will also be available on an open access basis to those interested in the detail of our methods and findings. Finally, to make relevant stakeholders aware 
of the 'headline' findings of the realist review, we will produce summaries in a user-friendly and tailored format.

\section{Author affiliations}

${ }^{1}$ Department of Public Health and Primary Care, University of Cambridge, Cambridge, UK

${ }^{2}$ Medical Library, School of Clinical Medicine, University of Cambridge, Cambridge, UK

${ }^{3}$ Institute Health and Society, Newcastle University, Newcastle upon Tyne, UK ${ }^{4} \mathrm{NHS}$ Cambridgeshire and Peterborough Clinical Commissioning Group, Cambridge, UK

${ }^{5}$ School of Medicine, Trinity College Dublin, Dublin, Ireland

${ }^{6}$ Population Health Sciences Institute, Newcastle University, Newcastle upon Tyne, UK

${ }^{7}$ Patient and Public Involvement Representative, Norfolk, UK

${ }^{8}$ Patient and Public Involvement Representative, Peterborough, UK

${ }^{9}$ Patient and Public Involvement Representative, Norwich, UK

${ }^{10}$ Nuffield Department of Primary Care Health Sciences, Oxford University, Oxford, UK

\section{Twitter Isla Kuhn @ilk21 and Sarah Sowden @SarahLSowden}

Contributors JAF: conceived the idea and drafted the initial funding application, from which manuscript is drawn, with input from GW, FH, SS, IK, CB, RRH, AM and SM. JAF, GW, FH, SS, IK, CB, RRH, AM, SM and RS: contributed to the design of the study after funding. AG: drafted the initial version of this manuscript and JAF, GW, $\mathrm{FH}, \mathrm{SS}$, IK, CB, RRH, AM, SM and RS: contributed to editing the final version. JAF is the guarantor.

Funding This study was supported by the National Institute for Health Research (NIHR) Health Services and Delivery Research Programme, grant number NIHR130694.

Competing interests GW is: deputy chair of the UK's National Institute for Health Research Health Technology Assessment Prioritisation Committee: Integrated Community Health and Social Care (A) and a member of the Methods Group (A).

Patient consent for publication Not required.

Provenance and peer review Not commissioned; peer reviewed for ethical and funding approval prior to submission.

Supplemental material This content has been supplied by the author(s). It has not been vetted by BMJ Publishing Group Limited (BMJ) and may not have been peer-reviewed. Any opinions or recommendations discussed are solely those of the author(s) and are not endorsed by BMJ. BMJ disclaims all liability and responsibility arising from any reliance placed on the content. Where the content includes any translated material, BMJ does not warrant the accuracy and reliability of the translations (including but not limited to local regulations, clinical guidelines, terminology, drug names and drug dosages), and is not responsible for any error and/or omissions arising from translation and adaptation or otherwise.

Open access This is an open access article distributed in accordance with the Creative Commons Attribution Non Commercial (CC BY-NC 4.0) license, which permits others to distribute, remix, adapt, build upon this work non-commercially, and license their derivative works on different terms, provided the original work is properly cited, appropriate credit is given, any changes made indicated, and the use is non-commercial. See: http://creativecommons.org/licenses/by-nc/4.0/.

\section{ORCID iDs}

John Alexander Ford http://orcid.org/0000-0001-8033-7081

Sarah Sowden http://orcid.org/0000-0001-9359-3463

Rikke Siersbaek http://orcid.org/0000-0003-3223-1420

\section{REFERENCES}

1 GBD 2017 Causes of Death Collaborators. Global, regional, and national age-sex-specific mortality for 282 causes of death in 195 countries and territories, 1980-2017: a systematic analysis for the global burden of disease study 2017. Lancet 2018;392:1736-88.

2 Mackenbach JP. Health inequalities - Persistence and change in European welfare states. Oxford University Press, 2019.

3 Bambra C, Riordan R, Ford J, et al. The COVID-19 pandemic and health inequalities. J Epidemiol Community Health 2020;74:jech2020-214401-8.
4 Institute for Health Equity. Social inequalities in the leading causes of early death a life course approach, 2015. Available: http://www. instituteofhealthequity.org/resources-reports/social-inequalities-inthe-leading-causes-of-early-death-a-life-course-approach/socialinequalities-in-the-leading-causes-of-early-death-a-life-courseapproach.pdf [Accessed 9 Jul 2019].

5 Office for National Statistics. Health state life expectancies by national deprivation deciles, England and Wales - Office for National Statistics, 2019. Available: https://www.ons.gov.uk/peoplepopulation andcommunity/healthandsocialcare/healthinequalities/bulletins/heal thstatelifeexpectanciesbyindexofmultipledeprivationimd/2015to2017 [Accessed 9 Jul 2019].

6 Public Health England. Health profile for England: 2018, 2018. Available: https://www.gov.uk/government/publications/healthprofile-for-england-2018 [Accessed 9 Jul 2019].

7 Buck D, Maguire D. Inequalities in life expectancy changes over time and implications for policy, 2015. Available: https://www.kingsfund. org.uk/sites/default/files/field/field_publication_file/inequalities-in-lifeexpectancy-kings-fund-aug15.pdf [Accessed 12 Jun 2019].

8 Forrest LF, Sowden S, Rubin G, et al. Socio-Economic inequalities in stage at diagnosis, and in time intervals on the lung cancer pathway from first symptom to treatment: systematic review and metaanalysis. Thorax 2017;72:430-6.

9 Lorenc T, Petticrew M, Welch V, et al. What types of interventions generate inequalities? Evidence from systematic reviews. $J$ Epidemiol Community Health 2013;67:190-3.

10 White M, Adams J, Heywood P. How and why do interventions that increase health overall widen inequalities within populations? Social inequality and public health Policy Press, 2009: 64-81.

11 McGill R, Anwar E, Orton L. Are interventions to promote healthy eating equally effective for all? systematic review of socioeconomic inequalities in impact health behavior, health promotion and society. BMC Public Health 2015;15.

12 Forrest LF, Adams J, Wareham H, et al. Socioeconomic inequalities in lung cancer treatment: systematic review and meta-analysis. PLoS Med 2013;10:e1001376.

13 Forrest LF, Adams J, Rubin G, et al. The role of receipt and timeliness of treatment in socioeconomic inequalities in lung cancer survival: population-based, data-linkage study. Thorax 2015;70:138-45.

14 Attwood S, van Sluijs E, Sutton S. Exploring equity in primary-carebased physical activity interventions using PROGRESS-Plus: a systematic review and evidence synthesis. Int $J$ Behav Nutr Phys Act 2016;13:60.

15 Durand M-A, Carpenter L, Dolan H, et al. Do interventions designed to support shared decision-making reduce health inequalities? A systematic review and meta-analysis. PLoS One 2014;9:e94670.

16 Tabuchi T, Iso H, Brunner E. Tobacco control measures to reduce socioeconomic inequality in smoking: the necessity, time-course perspective, and future implications. J Epidemiol 2018;28:170-5.

17 Asaria M, Doran T, Cookson R. The costs of inequality: wholepopulation modelling study of lifetime inpatient hospital costs in the English National health service by level of neighbourhood deprivation. J Epidemiol Community Health 2016;70:990-6.

18 NHS England. The NHS long term plan, 2019. Available: www. longtermplan.nhs.uk [Accessed 9 Jul 2019].

19 NHS England. Investment and evolution: a five-year framework for GP contract reform to implement the NHS long term plan, 2019. Available: https://www.england.nhs.uk/wp-content/uploads/2019/01/ gp-contract-2019.pdf [Accessed 9 Jul 2019].

20 Ford J, Sim F, Mackie P. Health inequalities: the need for clarity in the confusion. Public Health 2019;175:A1-2.

21 Vilhelmsson A, Östergren P-O. Reducing health inequalities with interventions targeting behavioral factors among individuals with low levels of education - A rapid review. PLoS One 2018;13:e0195774.

22 Arblaster L, Lambert M, Entwistle V, et al. A systematic review of the effectiveness of health service interventions aimed at reducing inequalities in health. J Health Serv Res Policy 1996;1:93-103 http:// www.ncbi.nlm.nih.gov/pubmed/10180855

23 O'Neill J, Tabish H, Welch V, et al. Applying an equity lens to interventions: using progress ensures consideration of socially stratifying factors to illuminate inequities in health. $J$ Clin Epidemiol 2014;67:56-64.

24 Terens N, Vecchi S, Bargagli AM, et al. Quality improvement strategies at primary care level to reduce inequalities in diabetes care: an equity-oriented systematic review. BMC Endocr Disord 2018;18:31

25 Capewell S, Graham H. Will cardiovascular disease prevention widen health inequalities? PLoS Med 2010;7:e1000320.

26 Bambra C, Gibson M, Sowden A, et al. Tackling the wider social determinants of health and health inequalities: evidence from systematic reviews. J Epidemiol Community Health 2010;64:284-91. 
27 Adams J, Mytton O, White M, et al. Why are some population interventions for diet and obesity more equitable and effective than others? the role of individual agency. PLoS Med 2016;13:e1001990.

28 Greenhalgh T, Peacock R. Effectiveness and efficiency of search methods in systematic reviews of complex evidence: audit of primary sources. BMJ 2005;331:1064-5.

29 Ramalho A, Castro P, Gonçalves-Pinho M, et al. Primary health care quality indicators: an umbrella review. PLoS One 2019;14:e0220888.

30 Booth A, Clarke M, Ghersi D, et al. An international registry of systematic-review protocols. Lancet 2011;377:108-9.

31 Emmel N, Greenhalgh J, Manzano A. Data Gathering in Realist Reviews Looking for needles in haystacks. In: Doing realist research. SAGE Publications Ltd, 2019: 131-46.
32 Wong G, Greenhalgh T, Westhorp G, et al. Development of methodological guidance, publication standards and training materials for realist and meta-narrative reviews: the RAMESES (realist and Meta-narrative evidence syntheses - evolving standards) project. Heal Serv Deliv Res 2014;2:1-252.

33 NHS England. Improving access for all: reducing inequalities in access to general practice services, 2017. Available: https://www. england.nhs.uk/publication/improving-access-for-all-reducinginequalities-in-access-to-general-practice-services/ [Accessed 23 Apr 2021]. 